\title{
Lgr5 in cancer biology: functional identification of Lgr5 in cancer progression and potential opportunities for novel therapy
}

\author{
Liangliang $\mathrm{Xu}^{1,2+}$, Weiping Lin $\mathrm{Li}^{3,4+}$, Longping Wen ${ }^{5^{*}}$ and Gang $\mathrm{Li}^{3,4,6^{*}}$ (D)
}

\begin{abstract}
Cancer remains one of the leading lethal diseases worldwide. Identifying biomarkers of cancers might provide insights into the strategies for the development of novel targeted anti-cancer therapies. Leucine-rich repeat-containing $G$ proteincoupled receptor 5 ( $\mathrm{Lgr} 5)$ has been recently discovered as a candidate marker of cancer stem cell populations. Aberrant increased expression of Lgr5 may represent one of the most common molecular alterations in some human cancers, leading to long-term potentiation of canonical Wnt/ $\beta$-catenin signaling. On the other hand, however, Lgr5-mediated suppression in canonical Wnt/ $\beta$-catenin signaling has also been reported in certain cancers, such as B cell malignancies. Until now, therapeutic approaches targeting Lgr5-associated signaling axis are not yet clinically available. Increasing evidence have indicated that endogenous $\mathrm{Lgr}^{+}$cell population is implicated in tumor initiation, progression, and metastasis. This review is to summarize our current knowledge about the importance of Lgr5 in cancer biology and the underlying molecular mechanisms of Lgr5-mediated tumor-promoting/suppressive activities, as well as potentially useful preventive strategies in treating tumor. Therefore, targeted therapeutic modulation of $\mathrm{Lgr}^{+}$cancer cell population by targeting Wnt/ $\beta$-catenin signaling through targeted drug delivery system or targeted genome editing might be promising for potential novel anti-cancer treatments. Simultaneously, combination of therapeutics targeting both Lgr $5^{+}$ and Lgr5 ${ }^{-}$cancer cells may deserve further consideration considering the plasticity of cancer cells. Also, a more specific targeting of cancer cells using double biomarkers may be much safer and more effective for anti-cancer therapy.
\end{abstract}

Keywords: Lgr5, Wnt/ß-catenin signaling, Cancer, Metastasis

\section{Introduction}

Cancer remains among the most challenging diseases worldwide although extensive studies have been performed and novel systemic treatment advances during recent decades. In general, most cancer deaths are attributed to tumor recurrence and metastasis $[1,2]$. Therefore, it is necessary and imperative to better understand the cellular and molecular mechanisms underlying cancer recurrence and tumor metastasis.

\footnotetext{
* Correspondence: wenlp@scut.edu.cn; gangli@cuhk.edu.hk

${ }^{+}$Liangliang Xu and Weiping Lin contributed equally to this work.

${ }^{5}$ Nanobio Laboratory, Institute of Life Sciences, South China University of

Technology, Guangzhou, Guangdong, People's Republic of China

${ }^{3}$ Department of Orthopaedics and Traumatology, Faculty of Medicine, Prince of Wales Hospital, The Chinese University of Hong Kong, Shatin, Hong Kong SAR PRC

Full list of author information is available at the end of the article
}

Increasing evidence suggest that both intrinsic properties of cancer cells and host organ microenvironment participate actively in tumor metastasis [3]. The patterns of organ-specific metastasis of tumor are determined by the concept of "seed (the cancer cell) and soil (the secondary organ)," which was first proposed by Dr. Stephen Paget in $1889[4,5]$. Hence, the process of tumor progression has been regarded as a result of an evolving crosstalk between different cell types within the tumor and its surrounding host tissues and organs or tumor stroma [6-8]. It has been well documented that tumor sites are in a hypoxic and inflammatory microenvironment with the release of various chemokines, cytokines, and growth factors that recruit bone marrow-derived cells (e.g., mesenchymal stem cells, macrophages) into the tumor sites via interactions with the surface receptors of 
bone marrow-derived cells [9-11]. These factors secreted by tumor cells include interleukins, tumor necrosis factor$\alpha$ (TNF- $\alpha$ ), stromal cell-derived factor 1 (SDF-1), and other identified and unidentified inflammatory transmitters [12-15]. Also, the tumor microenvironment contains various inflammatory cells, including myeloid cell subpopulations, innate and adaptive immune cells $\mathrm{NK}(\mathrm{T})$ cells, and $B$ and $T$ cells $[16,17]$. Thus, this dynamic tumor microenvironment with hypoxia and inflammation may be responsible for tumor cell variants through genomic instability, genomic heterogeneity, and epigenetic alterations, making tumor unpredictably behavioral diversified and difficult to cure $[18,19]$.

It is generally believed that chronic inflammatory diseases are well-recognized causes of cancer, accounting for $20 \%$ of all cancer deaths worldwide [20-22]. A recent study reported that the development of a tumor-promoting immune environment in chronic inflammation was initiated through a regulatory $\mathrm{T}$ cell-dependent mechanism. Thus, interleukin-33 (IL-33)/regulatory $\mathrm{T}$ cells (Tregs) axis may become a potential therapeutic target for the treatment of chronic inflammation-associated cancers [21].

Tumor recurrence is also a great challenge for cancer therapy besides tumor invasion [23, 24], which may be attributed to abnormal immune response. Usually, a metastatic disease occurs after a prolonged period of dormancy, when disseminated cancer is present but clinically undetectable. It has been demonstrated that neutrophils recruited during lung inflammation could initiate the awakening of dormant cancer cells [25]. A recent study further discovered that neutrophil extracellular traps formed by neutrophils during lipopolysaccharide- or tobacco smoke-induced lung inflammation are required to awaken dormant cancer cells, causing tumor metastasis in mice consequently [26]. Therefore, the process of tumor progression behaves very much like an immune disease to some extent [27].

\section{Wnt signaling, Lgr5, and cancer}

Wnt signaling pathway has a crucial role in embryonic development, tissue regeneration, and diseases, in particular cancer [28-30]. The canonical $\mathrm{Wnt} / \beta$-catenin signaling is essential for regulating the stemness, proliferation, and differentiation of several adult stem cell niches, such as hair follicles in the skin, the intestinal crypt, the mammary gland, and the hematopoietic tissues [31-36]. In the meanwhile, however, canonical Wnt signaling is also implicated in the formation and progression of various human cancers [30, 37-39].

Leucine-rich repeat-containing $G$ protein-coupled receptor 5 (Lgr5), also known as G protein-coupled receptor 49 (GPR49), is an "orphan" receptor belonging to the G protein-coupled receptor (GPCR) family [40, 41]. Lgr5 was originally discovered and cloned by Dr. Aaron J. W.
Hsueh's group in 1998 [42]. The Lgr5 gene is $\sim 144 \mathrm{~kb}$ long and is located on chromosome 12 at position $12 \mathrm{q} 22-\mathrm{q} 23$. And its protein structure has been presented in Fig. 1 [43]. Accumulating body of evidence have indicated that Lgr5 is essential for normal embryonic development and emerges as a novel bona fide marker of adult stem cells in various organs and tissues exhibiting multi-biologic functions [34, 44-54].

Notably, Lgr5 has been demonstrated upregulated in various cancer tissues, such as basal cell carcinomas, hepatocellular carcinomas, colorectal tumors, and ovarian tumors $[55,56]$. In general, Lgr5 modulates canonical Wnt signaling strength through binding to its ligand Rspondin $[41,57]$. Lgr5 potentiates Wnt/ $\beta$-catenin signaling pathway, thereby stimulating cancer stem cell proliferation and self-renewal $[58,59]$. Lgr5 has been demonstrated to promote cancer cell mobility, tumor formation, and epithelial-mesenchymal transition in breast cancer cells via activation of Wnt/ $\beta$-catenin signaling. Notably, Lgr5 is required for the maintenance of breast cancer stem cells [58]. Furthermore, positive correlations between high expression of Lgr 5 and shorter survival of patients have been reported [2]. Studies have further demonstrated that Lgr5 regulates the malignant phenotype in a subset of patientderived glioblastoma stem cells, which may represent as a potential predictive marker of glioblastoma [60]. On the other hand, however, Lgr5 have been shown to negatively regulate $\mathrm{Wnt} / \mathrm{\beta}$-catenin signaling in some special occasions [61]. Importantly, numerous studies using genetic lineage tracing analysis or detection by antibodies against Lgr5 have indicated Lgr 5 as biomarkers of cancer stem cells of various human cancer types, such as adenocarcinoma, glioblastoma, and colorectal and breast cancers [62-71].

Interestingly, canonical and non-canonical Wnt signaling pathways seem to exhibit opposing effects on tumor growth [72-75]. The canonical Wnt signaling stimulates liver growth and regeneration [76], and is reported to be activated in "well-differentiated" hepatocellular carcinomas cell subtypes but is repressed in "poorly differentiated" subtypes [73, 77]. Also, potentiated canonical Wnt signaling may contribute to glioblastoma cell growth through maintaining cancer stemness trait and stimulating cancer metastasis [75]. In contrast, activation of non-canonical Wnt signaling has been demonstrated to inhibit tumor growth [73, 74, 78], possibly mediated by antagonizing canonical Wnt signaling [73].

\section{Lgr5 in malignant hematopoiesis}

Lgr5, a Wnt target gene, has been widely used as a marker of organ stem cells with self-renewal capacity [41, 79], as well as an established biomarker of cancer stem cells (e.g., colorectal cancer and mammary tumors) [80]. Simultaneously, Lgr5 has been recently reported to be essential for B cell development. Self-renewal in the B 

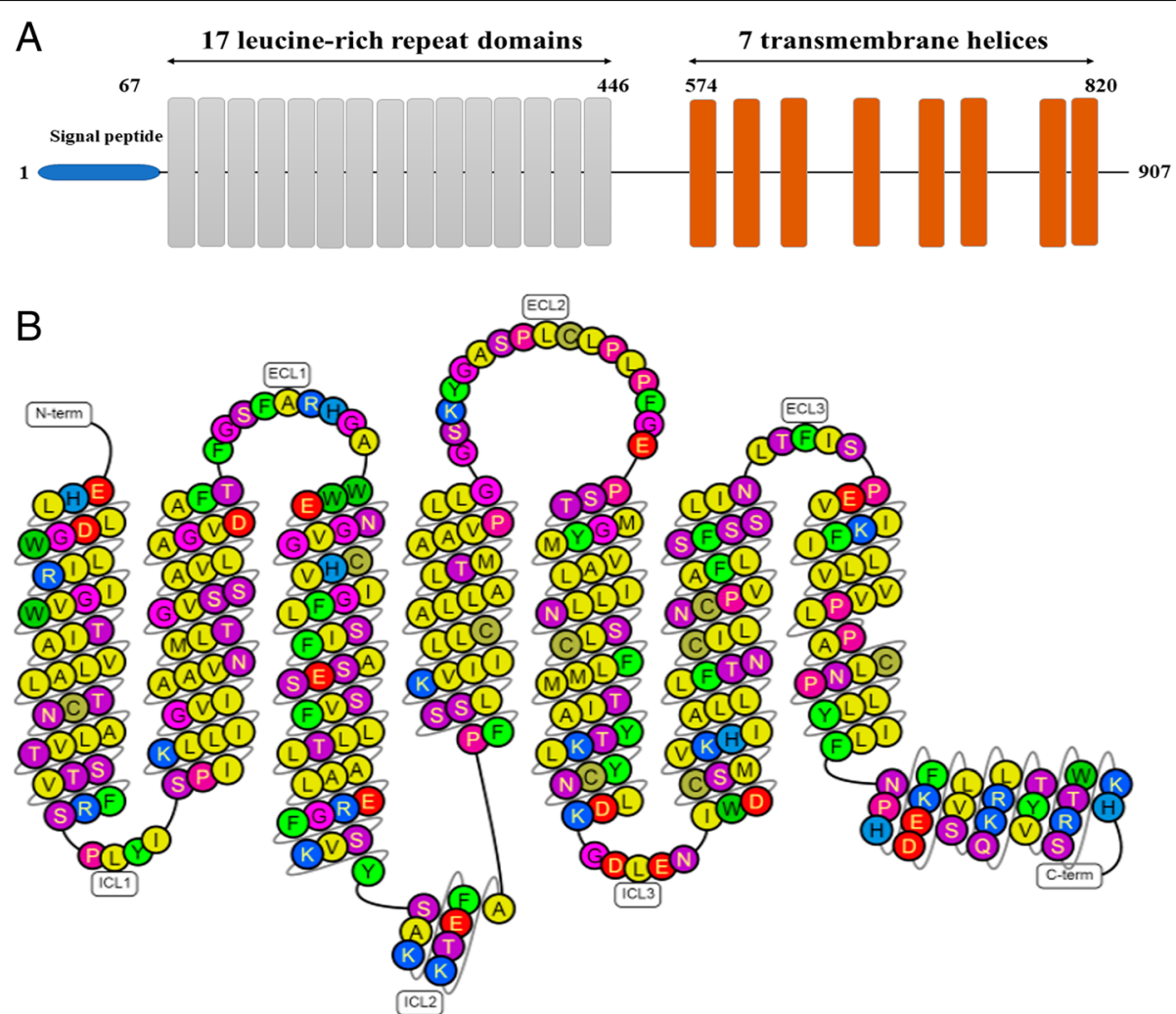

Fig. 1 The schematic illustration of the general structure of Lgr5. a Lgr5 comprises of a signal peptide (blue) followed by 17 leucine-rich repeat (LRR) domains (gray). Also, it contains a linker region between the last LRR and the first transmembrane (TM) domain, followed by a seven helical TM domain homologs to rhodopsin-like G protein receptors (GPCRs). $\mathbf{b}$ The diagram showing the structure of human Lgr5 is produced by GPCRdb (http://docs.gpcrdb.org/index.html). ICL, intracellular loops; ECL, extracellular loops

cell lineage is induced by positive selection and antigenreceptor (BCR) signaling, i.e., an encounter of cognate antigen. Self-renewal at this stage leads to clonal expansion and survival. Studies have demonstrated Lgr5 expression in B cells, which restricts the levels of nuclear $\beta$-catenin and enables $B$ cell survival through negative regulation of canonical Wnt-signaling, although most studies suggest the potentiated role of Lgr5 in the regulation of Wnt signaling [81]. Thus, Lgr5 enables positive B cell selection and tumor initiation in B cell malignancies [82]. Lgr5 may become a potential diagnostic marker and therapeutic target for B cell malignancies.

\section{Targeting $\operatorname{Lgr} 5^{+}$cancer stem cells}

Cancer stem cells (CSCs) have been identified as a driving force in tumor initiation, growth, and metastases [83, 84]. Also, CSCs are believed to play central roles in drug resistance and resistance of standard chemotherapy and radiation treatment [85-88]. Cancer cells in primary solid tumors reside in a complex microenvironment comprising numerous cell types, including endothelial cells of the blood vessels, lymphatics, pericytes, adipocytes, and various bone marrow-derived cells, such as macrophages, platelets, and mesenchymal stem cells [89-91].
Despite stem cell niches of different tissues and species share many structural similarities, they differ in their functional characteristics [92]. Many CSC biomarkers are emerging as prognostic factors for carcinogenesis, tumor aggressiveness and, therapeutic resistance. Studies have demonstrated that CSCs could be characterized using markers, such as CD133 [83, 93], CD24/29 [94, 95], or a combination of CD44 and CD166 [96]. Strikingly, Lgr5 has been referred to as a novel biomarker of various human cancers of the stomach [97], pancreas [98, 99], liver [100], colon [101], and ovary [102] during recent years. Notably, CSCs are critical for the formation and maintenance of tumor growth and metastasis, which may represent a therapeutic opportunity for cancer therapy. However, until now, whether a single CSC-targeting therapy is sufficient to eradicate cancers still remains controversial, considering the potential treatment evasion by non-CSC plasticity [103].

Enhanced Wnt signaling was detected in CSCs due to the elevated $\beta$-catenin localization within the nuclear region. Interestingly, Lgr5, a cell surface-expressed Wnt target gene, has been demonstrated as a functional biomarker of cancer stem cells, contributing to cancer stem cell proliferation and self-renewal through the regulation 
of Wnt $/ \beta$-catenin signaling pathway. Notably, the expression of Lgr5 is reported to be increased in human colorectal adenomas and cancers [104-106], hepatocellular carcinoma, basal cell carcinoma, and neuroblastoma [55, 107, 108]. Lgr $^{+}$cells have been demonstrated to be the cells of origin of tumors [109], which may provide a feasible approach for effective targeted anti-tumor treatment through targeted elimination of $\mathrm{Lgr}^{+}$CSCs selectively.

A recent study reported that targeting $\mathrm{Lgr}^{+}$cells with an antibody conjugated to distinct drugs exhibited potent efficacy to decrease tumor size and proliferation of colon cancer [110]. Simultaneously, a similar study further confirmed the tumor eradicative and recurrencepreventive effects through Lgr5-targeted antibody-drug conjugates in a xenograft model of colon cancer [111]. Furthermore, $\operatorname{Lgr}^{+}{ }^{+} \mathrm{CSC}$-targeted drug delivery system might also become a promising approach for targeted anti-tumor therapy. A recent study reported that RSPO1 (a Lgr5 natural ligand)-conjugated liposomes encapsulating doxorubicin led to massive tumor tissue necrosis and growth inhibition through efficient targeting of Lgr $5^{+}$CSCs in a patient-derived xenograft tumor model [112]. However, studies have also shown that selective ablation of $\mathrm{Lgr}^{+}$CSCs using gene-specific approach is effective temporarily yet is eventually overcome by robust plasticity of non-targeted cancer cells [65]. Hence, the reappearance of $\mathrm{Lgr}^{+}$cells following complete elimination of the $\operatorname{Lgr} 5^{+}$colorectal cancers may result from the plasticity of $\operatorname{Lgr}^{-}$colorectal cancers and the transition between $\mathrm{Lgr}^{+}$CSCs and $\mathrm{Lgr5}^{-}$CSCs [66]. Hence, accumulating preclinical evidence have suggested the potential of targeting Lgr5 via Lgr5-targeted antibody-drug conjugates as effective novel therapeutics for tumor treatment [110,111, 113]. A single targeting of $\mathrm{Lgr}^{+}$cancer cells may not be able to eradicate cancers and prevent tumor recurrence sufficiently for some certain cancer types at a specific stage. Furthermore, a combined therapy targeting both $\mathrm{Lgr} 5^{+}$and $\mathrm{Lgr} 5^{-}$cancer cells may desire further consideration and experimental validation.

In addition, drug screening targeting Lgr5-associated signaling pathways in tumor organoids ex vivo might provide a powerful tool for the development of novel drugs for personalized anti-tumor therapy [114-119]. Also, Lgr5, a surface-expressed protein, may become a potential therapeutic target for tumor therapy through antibodybased or drug delivery therapies. Simultaneously, more importantly, more comprehensive studies on the plasticity of cancer cells within a solid tumor are warranted to the recurrence of $\mathrm{Lgr}^{+}$cells following targeted elimination of $\mathrm{Lgr}^{+}$cancer cells, which may be instrumental for the development of novel strategies for more effective antitumor treatments.

\section{Multifaceted roles of Lgr5 during cancer progression}

Generally, numerous reports have indicated that Lgr5 promotes both development and survival of various cancer types through potentiation of $\mathrm{Wnt} / \beta$-catenin signaling, such as colorectal cancer, and glioblastomas [120]. Also, studies have demonstrated close associations between Lgr5 and aggressiveness in neuroblastoma cell lines at different stages of treatment [121], as well as in papillary thyroid cancer [122]. Further studies have indicated the involvement of Lgr5 in drug resistance through Wnt signaling in neuroblastoma cell lines [120]. Therefore, increasing evidence suggest that targeted suppression of Lgr5 in some certain cancer types may represent potential therapeutic approaches for treating cancer.

However, until now, the prognostic significance of Lgr5 in cancer still remains controversial. Lgr5 may exert multiple or even different functions between different cancer types. Discrepant results of reports on the effects of Lgr5 expression in tumor progression have been presented in the literature. Reports of Lgr5-mediated tumor growth in human glioma have been listed in Table 1, whilst studies on Lgr5-induced tumor suppression in human colorectal cancer listed in Table 2. Numerous studies have reported the stimulatory effects of Lgr5 in tumor growth through the regulation of CSC stemness, epithelial-mesenchymal transition (EMT), and cancer cell proliferation (Fig. 2) $[58,108,123$, 127].

Studies have shown that Lgr5 is implicated in tumor growth and metastasis via various signaling pathways.

Table 1 Lgr5-mediated stimulation of tumor growth in glioma

\begin{tabular}{|c|c|c|c|}
\hline Authors & Tumor models & Conclusions & Mechanisms \\
\hline $\begin{array}{l}\text { Vieira } \\
\text { et al. } \\
{[108]}\end{array}$ & $\begin{array}{l}\text { Neuroblastoma cell lines } \\
\text { (SK-N-BE(2)-C, IMR32, NGP, } \\
\text { SH-SY5Y, GIMEN, and SK-N-NAS) }\end{array}$ & $\begin{array}{l}\text { Lgr5 stimulated tumor growth and } \\
\text { proliferation }\end{array}$ & $\begin{array}{l}\text { Regulation of MEK/ERK and Akt pro-survival } \\
\text { signaling pathways }\end{array}$ \\
\hline $\begin{array}{l}\text { Zhang } \\
\text { et al. } \\
{[123]}\end{array}$ & $\begin{array}{l}\text { Adult glioma patients; primary } \\
\text { glioma cells }\end{array}$ & $\begin{array}{l}\text { Lgr5 is a new functional glioma stem } \\
\text { cell (GSC) marker involved in EMT and } \\
\text { prognostic indicator of glioma }\end{array}$ & $\begin{array}{l}\text { Stimulation of EMT by activating the } \\
\text { Wnt/ } \beta \text {-catenin pathway and maintenance } \\
\text { of GSC stemness by modulating the expression } \\
\text { of SOX2, Nanog, CD133, CD } 44, \text { CD24, and EpCAM }\end{array}$ \\
\hline $\begin{array}{l}\text { Xie et } \\
\text { al. [60] }\end{array}$ & $\begin{array}{l}\text { Glioblastoma patient-derived stem } \\
\text { cell (GSC) cultures }\end{array}$ & $\begin{array}{l}\text { Stimulation of tumorigenicity and } \\
\text { invasion by Lgr5 }\end{array}$ & $\begin{array}{l}\text { Regulation of the interactions between tumor cells and their } \\
\text { microenvironment through extracellular matrix and collagen } \\
\text { signaling pathways }\end{array}$ \\
\hline
\end{tabular}


Table 2 Lgr5-mediated tumor suppression in colorectal cancer

\begin{tabular}{llll}
\hline Authors & Tumor models & Conclusions & Mechanisms \\
\hline Jang et al. [124] & $\begin{array}{l}\text { Colorectal cancer (CRC) samples } \\
\text { collected from patients }\end{array}$ & $\begin{array}{l}\text { Lgr5 functions as a tumor suppressor in } \\
\text { the late stages of CRC progression }\end{array}$ & $\begin{array}{l}\text { Attenuation of proliferation, migration, and } \\
\text { colony formation of colon cancer cells }\end{array}$ \\
Zhou et al. [125] & $\begin{array}{l}\text { Human colon carcinoma cell lines } \\
\text { (HCT116, RKO, FET, CBS, HCT116b, }\end{array}$ & $\begin{array}{l}\text { Lgr5 inhibited cell survival and clonogenic } \\
\text { of CRC in vitro; suppression of metastasis } \\
\text { and TENN) }\end{array}$ & $\begin{array}{l}\text { R-spondin 1/Lgr5-induced activation of } \\
\text { TGF }-\beta \text { signaling with TGF- } \beta \text { type II receptor, } \\
\text { inducing inhibition and apoptosis of CRC }\end{array}$ \\
& $\begin{array}{ll}\text { Primary colorectal tumors from CRC } \\
\text { Wu [126] }\end{array}$ & Inhibition of tumor growth of CRC & $\begin{array}{l}\text { RSPO2-induced, Lgr5-dependent Wnt } \\
\text { signaling-negative feedback loop }\end{array}$ \\
& DLD1, HT29, and HCT116) & & \\
& & & \\
\hline
\end{tabular}

For example, during the progression of neuroblastoma development, Lgr5 enhanced tumor survival and proliferation through the regulation of phosphorylation of mitogen/extracellular signal-regulated kinases (MEK1/2) and extracellular signal-regulated kinases (ERK1/2), besides through reinforcement of $\mathrm{Wnt} / \beta$-catenin signaling [108]. Additionally, nuclear IKB-kinase $\alpha$ (IKK $\alpha)$ could directly bind to the promoters of inflammation factors and LGR5, which upregulates Lgr5 expression in turn through the activation of STAT3 signaling pathway during cancer progression of basal cell carcinomas [128]. It is generally believed that the activation of nuclear factor
(NF) $-\mathrm{kB}$ inflammatory pathway leads to a pro-tumorigenic inflammatory microenvironment $[129,130]$. And the ІкBkinase complex (IKK $\alpha$ and IKK $\beta$ ) and its regulatory subunit $(\mathrm{IKK} \gamma)$ regulate the NF- $\mathrm{BB}$ signaling $[129,131]$. Thus, NF- $\mathrm{KB}$ signaling-induced abnormal immune response during cancer development may be another important stimulating factor of Lgr5 expression.

In contrast, a recent study has reported a suppressive role of Lgr5 during the late stages of colorectal cancer progression through attenuation of growth, colony formation, and migration capacities of colon cancer cells [124]. Also, Lgr5 is known to exhibit tumor-suppressive

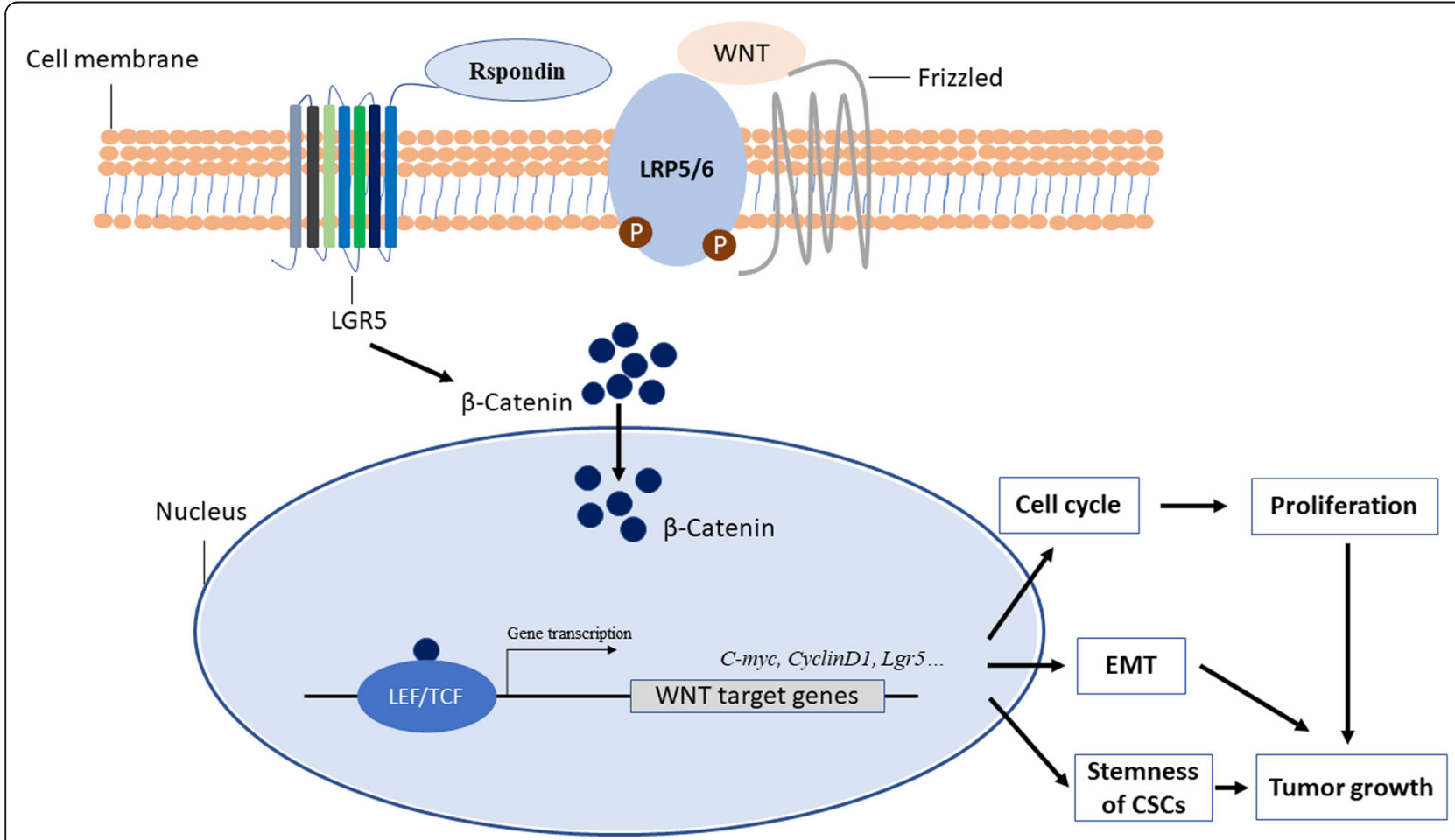

Fig. 2 Proposed model of the Lgr5-mediated stimulation of cancer growth through activation of the Wnt/ $\beta$-catenin signaling pathway. R-spondin activates Leucine-rich repeat-containing G protein-coupled receptor 5 (Lgr5). Once activated, Lgr5 protein recruits LRP-frizzled receptor complex, which binds to Wnt ligands, reinforcing Wnt signaling following phosphorylation of LRP5/6. A series of steps ensue, including the accumulation of $\beta$-catenin, which is translocated to the nucleus, inducing the expression of various Wht target genes (such as C-myc, Cyclin D1, Lgr5) after binding together with the TCF/LEF family of transcription factors, which leads to tumor progression through stimulation of cell proliferation, epithelial-mesenchymal-transition (EMT), and stemness maintenance of cancer stem cells (CSCS). TCF, T cell-factor; LEF, lymphoid enhancer-binding factor 
activity in the colon, which results from suppression of tumor growth and metastasis regulated by RSPO1/ Lgr5-mediated activation of TGF $\beta$ signaling [125]. Furthermore, studies have reported the RSPO2-induced, Lgr5-dependent Wnt signaling-negative feedback loop, exhibiting a tumor-suppressive activity in colorectal tumors [126]. Therefore, Lgr5 may exert complicated or even opposing functions during the progression of different cancer types.

\section{Potential crosstalk between $\operatorname{Lgr}^{+}{ }^{+}$stem cells and immune cells}

Inflammation has been shown as a hallmark of cancer development [132]. Chronic inflammation, which is caused by exposure to environmental agents, infection, genetic diseases, and metabolic disorders, is tightly correlated with various tumors, including lung carcinoma, gastric cancer, cervical cancer, colorectal cancer, hepatocellular carcinoma, and multiple myelomas [133, 134].

Resident immune cell populations of the mammalian innate immune system mainly comprise of macrophages, dendritic cells, and adaptive immune $T$ cells [135]. In particular, Tregs appear to be important regulators of adult stem cells under steady-state conditions [136]. Interestingly, Tregs have been demonstrated to preserve the integrity of $\mathrm{Lgr}^{+}$intestinal stem cells in the intestine, whilst depletion of Tregs would cause a dramatic reduction in the number of intestinal stem cells [137]. Also, co-culture of intestinal organoid cultures with Tregs or their effector cytokine IL-10 induced a rapid enrichment of $\mathrm{Lgr}^{+}$intestinal stem cell pool significantly ex vivo [137]. Therefore, interactions between $\mathrm{T}$ helper cells and $\mathrm{Lgr}^{+}$stem cells may probably take place during the progression of tumor-promoting immune microenvironment of chronic inflammation due to their proximity, which may play critical roles in tumor growth and metastasis.

\section{Safety concerns over Lgr5-targeted therapy}

Although previous studies have shown some therapeutic effects of Lgr5-targeted anti-cancer therapy, there still exist some safety issues due to the presence of Lgr5expressing adult stem cells in adult mammals [138-141]. Hence, considering the expression of Lgr5 in various normal adult tissues as a bona fide marker of adult stem cells, a more specific targeting using double biomarkers (such as Lgr5 and CD133) may exhibit much safer and tissue-specific targeting efficacy. In the meanwhile, targeted modulation of Lgr5 expression in solid tumors through targeted genome editing system or specific drug delivery system carrying natural compounds, engineered nanoparticles, or Chinese herb screened might become promising approaches and important research directions for targeted anti-cancer therapy.

\section{Conclusion}

Taken together, Lgr5 emerges as a potential surfaceexpressing biomarker for targeted anti-tumor therapy. Lgr5 is crucial for tumor initiation, progression, and metastasis through regulation of CSC function, Wnt/B-catenin signaling pathway, and various other signaling pathways. Lgr5 plays complicated and multifaceted roles during tumor progression. Lgr5-associated signaling pathways may play different or even opposing roles in different cancer types. Thus, targeted therapeutic modulation of Lgr5-associated signaling pathways may provide potential opportunities for anti-cancer therapy.

Single targeting $\mathrm{Lgr}^{+}$CSCs in solid cancer through antibody-conjugated drug delivery system exhibited some anti-cancer therapeutic effects. However, although selective targeting of $\operatorname{Lgr} 5^{+} \mathrm{CSCs}$ through genetic ablation is effective to treat tumor temporarily, the anti-tumor effects would be overcome by non-targeted cancer cells eventually. Thus, a combined therapy targeting both $\operatorname{Lgr} 5^{+}$and Lgr5 ${ }^{-}$cancer populations may deserve further consideration. Further comprehensive studies investigating the plasticity of cancer cells and transitions between Lgr $5^{-}$ and Lgr $5^{+}$cancer cells are necessary. Drug screening for ex vivo tumor organoids may be promising for the development of personalized drugs treating tumor. Meanwhile, a more specific targeting of Lgr5-expressing tumor cells using double biomarkers may deserve further consideration and elucidation.

\section{Abbreviations \\ CRC: Colorectal cancer; CSCs: Cancer stem cells; EMT: Epithelial-mesenchymal transition; GPCR: G protein-coupled receptor; GPR49: G protein-coupled re- ceptor 49; GSC: Glioma stem cell; IL-33: Interleukin-33; Lgr5: Leucine-rich repeat-containing G protein-coupled receptor 5; SDF-1: Stromal cell-derived factor 1; TNF-a: Tumor necrosis factor-a; Tregs: Regulatory $T$ cells}

\section{Acknowledgements}

We thank the SMART program, Lui Che Woo Institute of Innovative Medicine, and the Lui Che Woo Foundation Limited for their donation.

\section{Authors' contributions}

GL and L-PW formulated the idea and critically revised the manuscript. L-LX and W-PL co-wrote the manuscript. All authors read and approved the final manuscript.

\section{Funding}

This work was supported, in part, by grants from the Natural Science Foundation of China (81430049 and 81772322).

Availability of data and materials

Please contact the author for data requests.

Ethics approval and consent to participate

Not applicable.

Consent for publication

Not applicable.

Competing interests

The authors declare that they have no competing interests. 


\section{Author details}

'Key Laboratory of Orthopaedics and Traumatology, The First Affiliated Hospital of Guangzhou University of Chinese Medicine, Guangzhou University of Chinese Medicine, Guangzhou, People's Republic of China. ${ }^{2}$ Laboratory of Orthopaedics and Traumatology, Lingnan Medical Research Center, Guangzhou University of Chinese Medicine, Guangzhou, People's Republic of China. ${ }^{3}$ Department of Orthopaedics and Traumatology, Faculty of Medicine, Prince of Wales Hospital, The Chinese University of Hong Kong, Shatin, Hong Kong SAR PRC. ${ }^{4}$ Stem Cells and Regenerative Medicine Laboratory, Lui Che Woo Institute of Innovative Medicine, Li Ka Shing Institute of Health Sciences, The Chinese University of Hong Kong, Prince of Wales Hospital, Shatin, Hong Kong SAR PRC. ${ }^{5}$ Nanobio Laboratory, Institute of Life Sciences, South China University of Technology, Guangzhou, Guangdong, People's Republic of China. ${ }^{6}$ The CUHK-ACC Space Medicine Centre on Health Maintenance of Musculoskeletal System, The Chinese University of Hong Kong Shenzhen Research Institute, Shenzhen, People's Republic of China.

\section{Published online: 29 July 2019}

\section{References}

1. Chambers AF, Groom AC, MacDonald IC. Dissemination and growth of cancer cells in metastatic sites. Nat Rev Cancer. 2002;2(8):563-72.

2. Nakata S, et al. LGR5 is a marker of poor prognosis in glioblastoma and is required for survival of brain cancer stem-like cells. Brain Pathol. 2013;23(1):60-72.

3. Zhang $\mathrm{T}$, et al. Bone marrow-derived mesenchymal stem cells promote growth and angiogenesis of breast and prostate tumors. Stem Cell Res Ther. 2013;4:70.

4. Paget $\mathrm{S}$. The distribution of secondary growths in cancer of the breast. 1889. Cancer Metastasis Rev. 1989;8(2):98-101.

5. Paget S. Stephen Paget's paper reproduced from The Lancet, 1889. Cance Metastasis Rev. 1989:8(2):98-101.

6. Mbeunkui $F$, Johann DJ. Cancer and the tumor microenvironment: a review of an essential relationship. Cancer Chemother Pharmacol. 2009;63(4):571-82.

7. Lorusso G, Rugg C. The tumor microenvironment and its contribution to tumor evolution toward metastasis. Histochem Cell Biol. 2008;130(6):1091-103.

8. Bissell MJ, Radisky D. Putting tumours in context. Nat Rev Cancer. 2001; 1(1):46-54.

9. Lee WYW, et al. Immortalized human fetal bone marrow-derived mesenchymal stromal cell expressing suicide gene for anti-tumor therapy in vitro and in vivo. Cytotherapy. 2013;15(12):1484-97.

10. Vadhanraj $\mathrm{S}$, et al. Phase-I trial of a mouse monoclonal-antibody against Gd3 ganglioside in patients with melanoma - induction of inflammatory responses at tumor sites. J Clin Oncol. 1988;6(10):1636-48.

11. Wang QL, et al. Grapefruit-derived nanovectors use an activated leukocyte trafficking pathway to deliver therapeutic agents to inflammatory tumor sites. Cancer Res. 2015:75(12):2520-9.

12. Gao $\mathrm{H}$, et al. Activation of signal transducers and activators of transcription 3 and focal adhesion kinase by stromal cell-derived factor 1 is required for migration of human mesenchymal stem cells in response to tumor cellconditioned medium. Stem Cells. 2009:27(4):857-65.

13. Klopp $\mathrm{AH}$, et al. Tumor irradiation increases the recruitment of circulating mesenchymal stem cells into the tumor microenvironment. Cancer Res. 2007:67(24):11687-95.

14. Song C, et al. Thymidine kinase gene modified bone marrow mesenchymal stem cells as vehicles for antitumor therapy. Hum Gene Ther. 2011;22(4):439-49.

15. Xu WT, et al. Human mesenchymal stem cells (hMSCs) target osteosarcoma and promote its growth and pulmonary metastasis. Cancer Lett. 2009;281(1):32-41.

16. Coca $\mathrm{S}$, et al. The prognostic significance of intratumoral natural killer cells in patients with colorectal carcinoma. Cancer. 1997:79(12):2320-8.

17. Suen WCW, et al. Natural killer cell-based cancer immunotherapy: a review on 10 years completed clinical trials. Cancer Investig. 2018;36(8):431-57.

18. Bristow RG, Hill RP. Hypoxia, DNA repair and genetic instability. Nat Rev Cancer. 2008;8(3):180-92

19. Xiong L, et al. Aberrant enhancer hypomethylation contributes to hepatic carcinogenesis through global transcriptional reprogramming. Nat Commun. 2019:10:335

20. Karin M, Lawrence T, Nizet $\mathrm{V}$. Innate immunity gone awry: linking microbial infections to chronic inflammation and cancer. Cell. 2006; 124(4):823-35.
21. Ameri AH, et al. IL-33/regulatory $\mathrm{T}$ cell axis triggers the development of a tumor-promoting immune environment in chronic inflammation. Proc Natl Acad Sci U S A. 2019;116(7):2646-51.

22. Neurath MF, Finotto S. IL-6 signaling in autoimmunity, chronic inflammation and inflammation-associated cancer. Cytokine Growth Factor Rev. 2011; 22(2):83-9.

23. Seligson DB, et al. Global histone modification patterns predict risk of prostate cancer recurrence. Nature. 2005;435(7046):1262-6.

24. Linde N, Fluegen G, Aguirre-Ghiso JA. The relationship between dormant cancer cells and their microenvironment. Adv Cancer Res. 2016;132:45-71.

25. De Cock JM, et al. Inflammation triggers Zeb1-dependent escape from tumor latency. Cancer Res. 2016;76(23):6778-84.

26. Albrengues J, et al. Neutrophil extracellular traps produced during inflammation awaken dormant cancer cells in mice. Science. 2018;361(6409):1353.

27. Murakami K, Terakado Y, Barker N. Analysis of a mechanism that initiates stemness in inflammation-driven gastric cancer cells. Cancer Sci. 2018;109:421.

28. Reya T, Clevers H. Wnt signalling in stem cells and cancer. Nature. 2005; 434(7035):843-50.

29. Leucht $P$, et al. Wnt signaling and bone regeneration: Can't have one without the other. Biomaterials. 2019;196:46-50.

30. Yue ZY, et al. LGR4 modulates breast cancer initiation, metastasis, and cancer stem cells. FASEB J. 2018;32(5):2422-37.

31. Fodde $\mathrm{R}$, Brabletz T. Wnt/beta-catenin signaling in cancer stemness and malignant behavior. Curr Opin Cell Biol. 2007;19(2):150-8.

32. Barker $\mathrm{N}$, et al. Identification of stem cells in small intestine and colon by marker gene Lgr5. Nature. 2007;449(7165):1003-U1.

33. de Lau W, et al. Lgr5 homologues associate with Wnt receptors and mediate R-spondin signalling. Nature. 2011;476(7360):293-U57.

34. Liu DH, et al. Leucine-rich repeat-containing G-protein-coupled receptor 5 marks short-term hematopoietic stem and progenitor cells during mouse embryonic development. J Biol Chem. 2014;289(34):23809-16.

35. Haegebarth A, Clevers H. Wnt signaling, Lgr5, and stem cells in the intestine and skin. Am J Pathol. 2009;174(3):715-21.

36. de Lau W, et al. The R-spondin/Lgr5/Rnf43 module: regulator of Wnt signal strength. Genes Dev. 2014;28(4):305-16.

37. Klaus A, Birchmeier W. Wht signalling and its impact on development and cancer. Nat Rev Cancer. 2008;8(5):387-98.

38. Mohammed MK, et al. Wnt/beta-catenin signaling plays an everexpanding role in stem cell self-renewal, tumorigenesis and cancer chemoresistance. Genes Dis. 2016:3(1):11-40.

39. Leushacke M, Barker N. Lgr5 and Lgr6 as markers to study adult stem cell roles in self-renewal and cancer. Oncogene. 2012;31(25):3009-22.

40. McDonald $\mathrm{T}$, et al. Identification and cloning of an orphan $\mathrm{G}$ proteincoupled receptor of the glycoprotein hormone receptor subfamily. Biochem Biophys Res Commun. 1998;247(2):266-70.

41. Birchmeier W. Stem cells. Orphan receptors find a home. Nature. 2011; 476(7360):287-8.

42. Hsu SY, Liang SG, Hsueh AJW. Characterization of two LGR genes homologous to gonadotropin and thyrotropin receptors with extracellular leucine-rich repeats and a $\mathrm{G}$ protein-coupled, seven-transmembrane region. Mol Endocrinol. 1998:12(12):1830-45.

43. Kumar KK, Burgess AW, Gulbis JM. Structure and function of LGR5: an enigmatic G-protein coupled receptor marking stem cells. Protein Sci. 2014; 23(5):551-65.

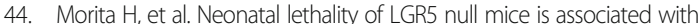
ankyloglossia and gastrointestinal distension. Mol Cell Biol. 2004;24(22):9736-43.

45. Garcia MI, et al. LGR5 deficiency deregulates Wnt signaling and leads to precocious Paneth cell differentiation in the fetal intestine. Dev Biol. 2009;331(1):58-67.

46. Jaks $V$, et al. Lgr5 marks cycling, yet long-lived, hair follicle stem cells. Nat Genet. 2008:40(11):1291-9.

47. Barker $\mathrm{N}$, et al. Lgr5(+ve) stem cells drive self-renewal in the stomach and build long-lived gastric units in vitro. Cell Stem Cell. 2010;6(1):25-36.

48. Barker N, Bartfeld S, Clevers H. Tissue-resident adult stem cell populations of rapidly self-renewing organs. Cell Stem Cell. 2010;7(6):656-70

49. Plaks $V$, et al. Lgr5-expressing cells are sufficient and necessary for postnatal mammary gland organogenesis. Cell Rep. 2013;3(1):70-8.

50. Huch $M$, et al. In vitro expansion of single Lgr5(+) liver stem cells induced by Wnt-driven regeneration. Nature. 2013;494(7436):247-50.

51. $\mathrm{Ng} \mathrm{A}$, et al. Lgr5 marks stem/progenitor cells in ovary and tubal epithelia. Nat Cell Biol. 2014;16(8):745-U342. 
52. Sun XF, et al. Ovarian LGR5 is critical for successful pregnancy. FASEB J. 2014;28(5):2380-9.

53. Boddupally K, et al. Lgr5 marks neural crest derived multipotent oral stromal stem cells. Stem Cells. 2016:34(3):720-31.

54. Lin W, et al. Lgr5-overexpressing mesenchymal stem cells augment fracture healing through regulation of Wnt/ERK signaling pathways and mitochondrial dynamics. FASEB J. 2019;33(7):8565-8577.

55. Tanese K, et al. G-protein-coupled receptor GPR49 is up-regulated in basal cell carcinoma and promotes cell proliferation and tumor formation. Am J Pathol. 2008; 173(3):835-43.

56. McClanahan T, et al. Identification of overexpression of orphan $\mathrm{G}$ proteincoupled receptor GPR49 in human colon and ovarian primary tumors. Cancer Biol Ther. 2006;5(4):419-26.

57. Carmon $\mathrm{KS}$, et al. R-spondins function as ligands of the orphan receptors LGR4 and LGR5 to regulate Wnt/beta-catenin signaling. Proc Natl Acad Sci U S A. 2011;108(28):11452-7.

58. Yang L, et al. LGR5 promotes breast cancer progression and maintains stem-like cells through activation of Wnt/beta-catenin signaling. Stem Cells. 2015;33(10):2913-24

59. Kemper $\mathrm{K}$, et al. Monoclonal antibodies against Lgr5 identify human colorectal cancer stem cells. Stem Cells. 2012:30(11):2378-86.

60. Xie Y, et al. LGR5 promotes tumorigenicity and invasion of glioblastoma stem-like cells and is a potential therapeutic target for a subset of glioblastoma patients. J Pathol. 2019;247(2):228-40.

61. Walker $F$, et al. LGR5 is a negative regulator of tumourigenicity, antagonizes Wnt signalling and regulates cell adhesion in colorectal cancer cell lines. PLoS One. 2011;6:e22733.

62. von Rahden BHA, et al. LgR5 expression and cancer stem cell hypothesis: clue to define the true origin of esophageal adenocarcinomas with and without Barrett's esophagus? J Exp Clin Cancer Res. 2011;141: 1762-72.

63. Liu SL, et al. Hedgehog signaling and Bmi-1 regulate self-renewal of normal and malignant human mammary stem cells. Cancer Res. 2006; 66(12):6063-71.

64. Kleist B, et al. Expression of the adult intestinal stem cell marker Lgr5 in the metastatic cascade of colorectal cancer. Int J Clin Exp Pathol. 2011;4(4):327-35.

65. Shimokawa M, et al. Visualization and targeting of LGR5(+) human colon cancer stem cells. Nature. 2017;545(7653):187.

66. Kobayashi S, et al. LGR5-positive colon cancer stem cells interconvert with drug-resistant LGR5-negative cells and are capable of tumor reconstitution. Stem Cells. 2012;30(12):2631-44.

67. Hirsch D, et al. LGR5 positivity defines stem-like cells in colorectal cancer. Carcinogenesis. 2013;35(4):849-58.

68. Hirsch D, et al. LGR5 positivity defines stem-like cells in colorectal cancer. Carcinogenesis. 2014;35(4):849-58.

69. Myssina, Svetlana, et al. Elevated expression of LGR5 and WNT signalling factors in neuroblastoma cells with acquired drug resistance. bioRxiv. 2018 1:449785. https://doi.org/10.1101/449785.

70. Shi S, et al. LGR5 acts as a target of miR-340-5p in the suppression of cell progression and drug resistance in breast cancer via Wnt/ $/$-catenin pathway. Gene. 2019;683:47-53.

71. Proctor $\mathrm{E}$, et al. Bmi1 enhances tumorigenicity and cancer stem cell function in pancreatic adenocarcinoma. PLoS One. 2013;8:e55820.

72. DeMorrow $\mathrm{S}$, et al. The endocannabinoid anandamide inhibits cholangiocarcinoma growth via activation of the noncanonical Wnt signaling pathway. Am J Physiol Gastrointest Liver Physiol. 2008;295(6): G1150-8.

73. Yuzugullu $\mathrm{H}$, et al. Canonical Wnt signaling is antagonized by noncanonical Wnt5a in hepatocellular carcinoma cells. Mol Cancer. 2009;8:90.

74. Toyama T, et al. Noncanonical Wnt11 inhibits hepatocellular carcinoma cell proliferation and migration. Mol Cancer Res. 2010;8(2):254-65.

75. Liu S, et al. R-spodin2 enhances canonical Wnt signaling to maintain the stemness of glioblastoma cells. Cancer Cell Int. 2018;18(1):156.

76. Apte $\mathrm{U}$, et al. Wnt/beta-catenin signaling mediates oval cell response in rodents. Hepatology. 2008;47(1):288-95.

77. Yang $\mathrm{W}$, et al. Wnt/beta-catenin signaling contributes to activation of normal and tumorigenic liver progenitor cells. Cancer Res. 2008;68(11):4287-95.

78. Proto, Maria Chiara, et al. Inhibition of Wnt/ $\beta$-Catenin pathway and Histone acetyltransferase activity by Rimonabant: a therapeutic target for colon cancer. Sci Rep. 2017;7(1):11678.

79. Van Der Flier LG, et al. The intestinal Wnt/TCF signature. Gastroenterology. 2007;132(2):628-32.
80. Nakata S, Phillips E, Goidts V. Emerging role for leucine-rich repeatcontaining G-protein-coupled receptors LGR5 and LGR4 in cancer stem cells. Cancer Manag Res. 2014;6:171-80.

81. Cosgun KNN, et al. Lgr5 functions as negative regulator of Wnt signaling in $B$ cells and is critical for self-renewal of normal and transformed B cells. Blood. 2017;130:3989.

82. Cosgun KN, et al. Lgr5 enables positive B-cell selection and tumor-initiation in B-cell malignancies. Blood. 2018;132:547. https://doi.org/10.1182/blood2018-99-116956

83. O'Brien CA, et al. A human colon cancer cell capable of initiating tumour growth in immunodeficient mice. Nature. 2007;445(7123):106-10.

84. Dick JE. Stem cell concepts renew cancer research. Blood. 2008;112(13): 4793-807.

85. Batlle E, Clevers H. Cancer stem cells revisited. Nat Med. 2017;23(10):1124-34.

86. Cojoc $\mathrm{M}$, et al. A role for cancer stem cells in therapy resistance: cellular and molecular mechanisms. Semin Cancer Biol. 2015;31:16-27.

87. Sun $\mathrm{R}$, et al. Nanoparticle-facilitated autophagy inhibition promotes the efficacy of chemotherapeutics against breast cancer stem cells. Biomaterials. 2016:103:44-55

88. Zhang $Y$, et al. Harnessing copper-palladium alloy tetrapod nanoparticleinduced pro-survival autophagy for optimized photothermal therapy of drug-resistant cancer. Nat Commun. 2018;9(1):4236.

89. Balkwill FR, Capasso M, Hagemann T. The tumor microenvironment at a glance. J Cell Sci. 2012;125(23):5591-6.

90. Luo Z, et al. Tumor microenvironment: the culprit for ovarian cancer metastasis? Cancer Lett. 2016;377(2):174-82.

91. Lin W, et al. Mesenchymal stem cells and cancer: clinical challenges and opportunities, vol. 2019; 2019.

92. Xia HM, et al. Tissue repair and regeneration with endogenous stem cells (vol 3, pg 174, 2018). Nat Rev Mater. 2018;3(8):279.

93. Todaro $\mathrm{M}$, et al. Colon cancer stem cells dictate tumor growth and resist cell death by production of interleukin-4. Cell Stem Cell. 2007;1 (4):389-402.

94. Vermeulen $L$, et al. Single-cell cloning of colon cancer stem cells reveals a multi-lineage differentiation capacity. Proc Natl Acad Sci U S A. 2008;105(36): $13427-32$.

95. Tirino V, et al. Cancer stem cells in solid tumors: an overview and new approaches for their isolation and characterization. FASEB J. 2013;27(1): 13-24.

96. Dalerba P, et al. Phenotypic characterization of human colorectal cancer stem cells. Proc Natl Acad Sci U S A. 2007;104(24):10158-63.

97. Wang $\mathrm{K}$, et al. Whole-genome sequencing and comprehensive molecular profiling identify new driver mutations in gastric cancer. Nat Genet. 2014; 46(6):573-82.

98. Wu J, et al. Whole-exome sequencing of neoplastic cysts of the pancreas reveals recurrent mutations in components of ubiquitin-dependent pathways. Proc Natl Acad Sci U S A. 2011;108(52):21188-93.

99. Jiang XM, et al. Inactivating mutations of RNF43 confer Wnt dependency in pancreatic ductal adenocarcinoma. Proc Natl Acad Sci U S A. 2013;1 10(31): 12649-54.

100. Ong CK, et al. Exome sequencing of liver fluke-associated cholangiocarcinoma. Nat Genet. 2012;44(6):690-U113.

101. Seshagiri S, et al. Recurrent R-spondin fusions in colon cancer. Nature. 2012; 488(7413):660

102. Ryland GL, et al. RNF43 is a tumour suppressor gene mutated in mucinous tumours of the ovary. J Pathol. 2013;229(3):469-76.

103. Kaiser J. The cancer stem cell gamble. Science. 2015;347(6219):226-9.

104. Fan XS, et al. Expression of Lgr5 in human colorectal carcinogenesis and its potential correlation with beta-catenin. Int J Color Dis. 2010;25(5):583-90.

105. Becker L, Huang Q, Mashimo H. Immunostaining of Lgr5, an intestinal stem cell marker, in normal and premalignant human gastrointestinal tissue. Thescientificworldjournal. 2008:8:1168-76.

106. Uchida $\mathrm{H}$, et al. Overexpression of leucine-rich repeat-containing $\mathrm{G}$ protein-coupled receptor 5 in colorectal cancer. Cancer Sci. 2010;101(7): $1731-7$.

107. Yamamoto Y, et al. Overexpression of orphan G-protein-coupled receptor, Grr49, in human hepatocellular carcinomas with beta-catenin mutations. Hepatology. 2003;37(3):528-33.

108. Vieira GC, et al. LGR5 regulates pro-survival MEK/ERK and proliferative Wnt/ beta-catenin signalling in neuroblastoma. Oncotarget. 2015;6(37):40053-67.

109. Barker N, et al. Crypt stem cells as the cells-of-origin of intestinal cancer. Nature. 2009;457(7229):608-U119. 
110. Junttila MR, et al. Targeting LGR5(+) cells with an antibody-drug conjugate for the treatment of colon cancer. Sci Transl Med. 2015;7(314):314ra186.

111. Gong X, et al. LGR5-targeted antibody-drug conjugate eradicates gastrointestinal tumors and prevents recurrence. Mol Cancer Ther. 2016; 15(7):1580-90.

112. Cao J, et al. Selective targeting and eradication of LGR5(+) thorn cancer stem cells using RSPO-conjugated doxorubicin liposomes. Mol Cancer Ther. 2018;17(7):1475-85.

113. Morgan RG, et al. Targeting LGR5 in colorectal cancer: therapeutic gold or too plastic? Br J Cancer. 2018;118(11):1410.

114. Thorne CA, et al. Small-molecule inhibition of Wnt signaling through activation of casein kinase 1 alpha. Nat Chem Biol. 2010;6(11):829-36.

115. Huang $L$, et al. Ductal pancreatic cancer modeling and drug screening using human pluripotent stem cell- and patient-derived tumor organoids. Nat Med. 2015;21(11):1364-71.

116. Skardal A, Shupe T, Atala A. Organoid-on-a-chip and body-on-a-chip systems for drug screening and disease modeling. Drug Discov Today. 2016; 21(9):1399-411.

117. Weeber $F$, et al. Tumor organoids as a pre-clinical cancer model for drug discovery. Cell Chem Biol. 2017;24(9):1092-100.

118. Verissimo CS, et al. Targeting mutant RAS in patient-derived colorectal cancer organoids by combinatorial drug screening. Elife. 2016;5:e18489.

119. Drost J, Clevers H. Organoids in cancer research. Nat Rev Cancer. 2018;18(7): 407-18.

120. Myssina S, et al. Elevated expression of LGR5 and WNT signalling factors in neuroblastoma cells with acquired drug resistance. bioRxiv. 2018;1:449785.

121. Forgham $\mathrm{H}$, et al. Stem cell markers in neuroblastoma-an emerging role for LGR5. Front Cell Dev Biol. 2015;3:77.

122. Michelotti G, et al. LGR5 is associated with tumor aggressiveness in papillary thyroid cancer. Oncotarget. 2015;6(33):34549-60.

123. Zhang J, et al. LGR5, a novel functional glioma stem cell marker, promotes EMT by activating the Wnt/beta-catenin pathway and predicts poor survival of glioma patients. J Exp Clin Cancer Res. 2018;37(1):225.

124. Jang $B G$, et al. Expression profile of LGR5 and its prognostic significance in colorectal cancer progression. Am J Pathol. 2018;188(10):2236-50.

125. Zhou XL, et al. R-spondin1/LGR5 activates TGF beta signaling and suppresses colon cancer metastasis. Cancer Res. 2017;77(23):6589-602.

126. Wu C, et al. RSPO2-LGR5 signaling has tumour-suppressive activity in colorectal cancer. Nat Commun. 2014;5:3149.

127. Chen Q, Cao HZ, Zheng PS. LGR5 promotes the proliferation and tumor formation of cervical cancer cells through the Wnt/beta-catenin signaling pathway. Oncotarget. 2014;5(19):9092-105.

128. Jia J, et al. LGR5 expression is controled by IKKalpha in basal cell carcinoma through activating STAT3 signaling pathway. Oncotarget. 2016;7(19):27280-94.

129. Perkins ND. Integrating cell-signalling pathways with NF-kappaB and IKK function. Nat Rev Mol Cell Biol. 2007:8(1):49-62.

130. Ye $X$, et al. Oncogenic potential of truncated RXRalpha during colitisassociated colorectal tumorigenesis by promoting IL-6-STAT3 signaling. Nat Commun. 2019;10(1):1463.

131. Hayden MS, Ghosh S. Shared principles in NF-kappaB signaling. Cell. 2008 132(3):344-62.

132. Hanahan D, Weinberg RA. Hallmarks of cancer: the next generation. Cell. 2011;144(5):646-74.

133. Grivennikov SI, Greten FR, Karin M. Immunity, inflammation, and cancer. Cell. 2010;140(6):883-99.

134. Elinav $E$, et al. Inflammation-induced cancer: crosstalk between tumours, immune cells and microorganisms. Nat Rev Cancer. 2013;13(11):759-71.

135. Fan X, Rudensky AY. Hallmarks of tissue-resident lymphocytes. Cell. 2016; 164(6):1198-211.

136. Burzyn D, Benoist C, Mathis D. Regulatory T cells in nonlymphoid tissues. Nat Immunol. 2013;14(10):1007-13.

137. Biton $\mathrm{M}$, et al. Thelper cell cytokines modulate intestinal stem cell renewal and differentiation. Cell. 2018;175(5):1307.

138. Barker N, Clevers H. Leucine-rich repeat-containing G-protein-coupled receptors as markers of adult stem cells. Gastroenterology. 2010;138(5): 1681-96.

139. Leung C, Tan SH, Barker N. Recent advances in Lgr5(+) stem cell research. Trends Cell Biol. 2018;28(5):380-91.

140. Lin W, et al. Characterisation of multipotent stem cells from human peripheral blood using an improved protocol. J Orthop Transl. 2019. In press
141. Huch M, Boj SF, Clevers H. Lgr5(+) liver stem cells, hepatic organoids and regenerative medicine. Regen Med. 2013;8(4):385-7.

\section{Publisher's Note}

Springer Nature remains neutral with regard to jurisdictional claims in published maps and institutional affiliations. 